\title{
Selective pyrolytic synthesis of anhydrothioglycosides of 1,2,4-triazoles
}

\author{
Ibtehal A. Al-Juwaiser, Elizabeth John, Maher R. Ibrahim, Yehia A. Ibrahim, \\ and Nouria A. Al-Awadi* \\ Chemistry Department, Faculty of Science, Kuwait University, P.O. Box 5969, Safat 13060, \\ Kuwait \\ E-mail:nouria@,kuc01.kuniv.edu.kw
}

\begin{abstract}
Presented at the $5^{\text {th }}$ Eurasian Meeting on Heterocyclic Chemistry EAMHC-5
(Kuwait, 1-6 March, 2008)
\end{abstract}

\begin{abstract}
The stereoselective pyrolytic conversion of the $2-(2,3,4,6$-tetra- $O$-acetyl- $\beta$-D- $N$-glucopyranosyl)1,2,4-triazole-3(4H)-thiones to the respective $5,2^{\prime}$-anhydro- $\beta$-D-mannopyranosides in moderate to good yields is described.
\end{abstract}

Keywords: Pyrolysis, nucleosides, anhydronucleosides, 1,2,4-triazoles

\section{Introduction}

Much attention has been directed to the synthesis of 2,2'- and 2,3'-anhydronucleosides owing to their ready attack by nucleophiles at the C-2' or C-3' positions, affording modified nucleoside derivatives with anti-AIDS activity. ${ }^{1}$ The reported method for the synthesis of 2,2'anhydronucleosides involved treating 1-(3',5'-O-isopropylidene-2'-O-methanesulfonyl- $\beta$-Dxylofuranosyl)-thymine with sodium hydroxide in refluxing ethanol to afford the corresponding 2,2'-anhydronucleoside. ${ }^{2}$ Recently, ${ }^{1 \mathrm{~d}, \mathrm{f}, \mathrm{g}, 3-5}$ the action of bases $\left(\mathrm{NaHCO}_{3} / \mathrm{DMF}, \mathrm{PhCOONa}\right.$ or $\mathrm{DBU}$ ) on the appropriate 2'-O-phenyloxycarbonyl or 2'-O-methanesulfonyl derivatives of nucleosides have been used to promote intramolecular cyclization. Additionally, heating 2'deoxy-2'-iodo-nucleosides in DMF with di- $n$-butyl-tin oxide gave the corresponding anhydronucleosides. $^{6}$ 2,2'-Anhydrothionucleosides have also been investigated and some synthetic methods have been reported. ${ }^{7 \mathrm{a}, \mathrm{b}}$ Moreover, a method has been reported for the synthesis of arabino-6-aza-2-thio-2,2' -anhydrouridine. ${ }^{8}$

The chemistry and diverse applications of heterocyclic glycosyl derivatives have received much attention, owing to their pronounced biological activities. Recently ${ }^{9}$ we reported a simple 
regioselective synthesis of 2-glycosyl derivatives of 1,2,4-triazine-3,5(2H,4H)-diones and their thiones (6-azauracil derivatives) which possess diverse biological activities such as cytotoxic, antiviral, enzyme inhibiting, immunosuppressive, antiphlogestic and bacteriostatic activities. ${ }^{10,11}$ Extension of the methodology enabled a direct stereoselective synthesis of 2- $N$-glycosyls of 1,2,4-triazole-3(2H)-thione which are of considerable biological interest. ${ }^{12}$ Scheme 1 illustrates the crucial step in our synthetic methods, which depends on selective protection-deprotection via the arylidene-amino group. In our previous work we have shown that removal of the benzonitrile derivatives leads to efficient selective synthesis of the desired 2-glycosyl derivatives 2 upon pyrolysis of the precursors arylidene-amino derivatives $\mathbf{1}$ (Scheme 1) at ca. 180-200 ${ }^{\circ} \mathrm{C}$. Kinetic studies of such conversions of $\mathbf{1}$ into $\mathbf{2}$ and their 1,2,4-triazole analogs proved that these pyrolytic conversions proceed via six-membered transition state involving concerted hetero-retro-ene eliminations. ${ }^{13}$

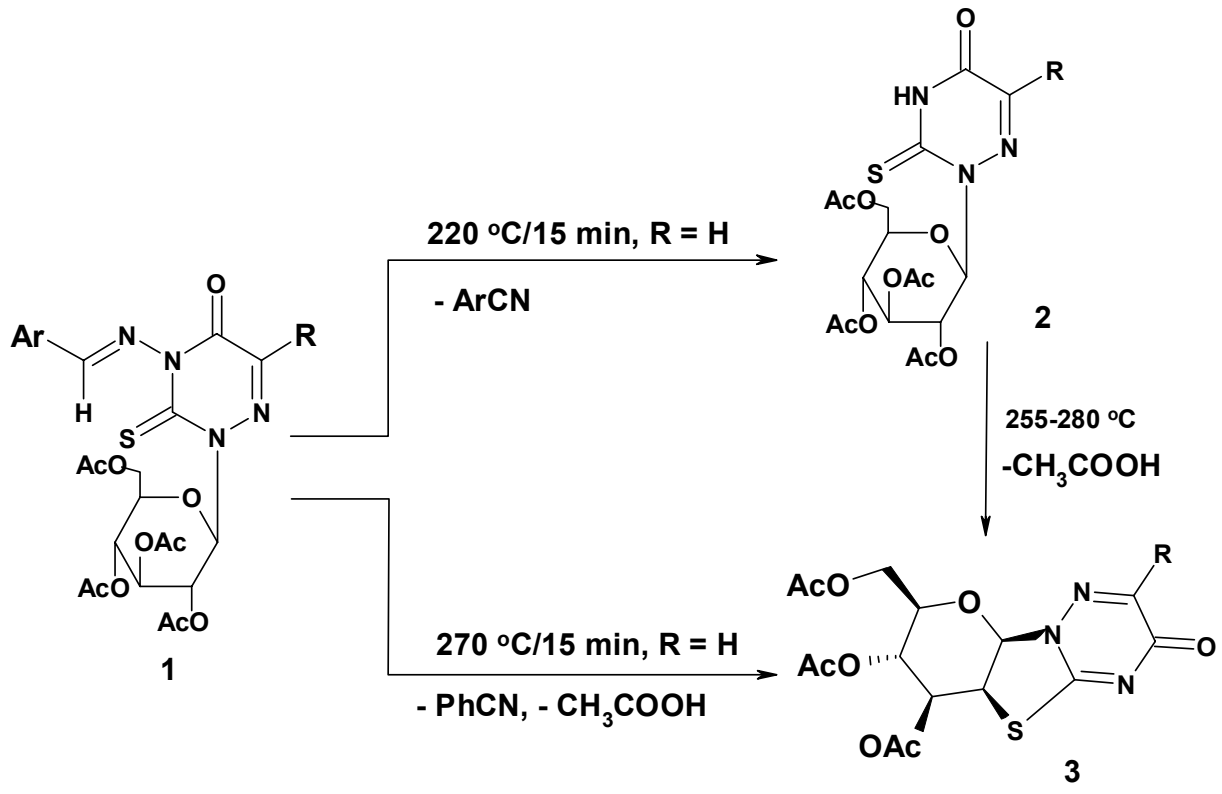

\section{Scheme 1}

During our kinetic studies of the pyrolysis of the 2-glucosyl-4-arylideneamino-3-thioxo-2,3dihydro-1,2,4-triazin-4(5H)-ones, $\mathbf{1}$, we found that heating 1 at a temperature above $220^{\circ} \mathrm{C}$ led to the formation of the expected $N$-glucosyl derivatives $\mathbf{2}$ in addition to a new derivative which was identified as the 3,2'-anhydro-2(3,4,6-tri- $O$-acetyl- $\beta$-D-mannosyl)-3-mercapto-1,2,4-triazin$5(2 \mathrm{H})$-one, 3. The yield of the latter was optimized at $255-280^{\circ} \mathrm{C}$ (depending on the Rsubstituents). ${ }^{14}$ These findings point to an interesting novel facile pyrolytic synthetic access to anhydroglycosyl derivatives. This gas-phase pyrolytic approach has also been extended to the synthesis of 3,2'-anhydro- $\beta$-D-talosyl, 5, and 3,2'-anhydro- $\beta$-D-arabinosyl, 7, derivatives of 3mercapto-1,2,4-triazin-5(2H)-ones (Scheme 2). ${ }^{14}$ 


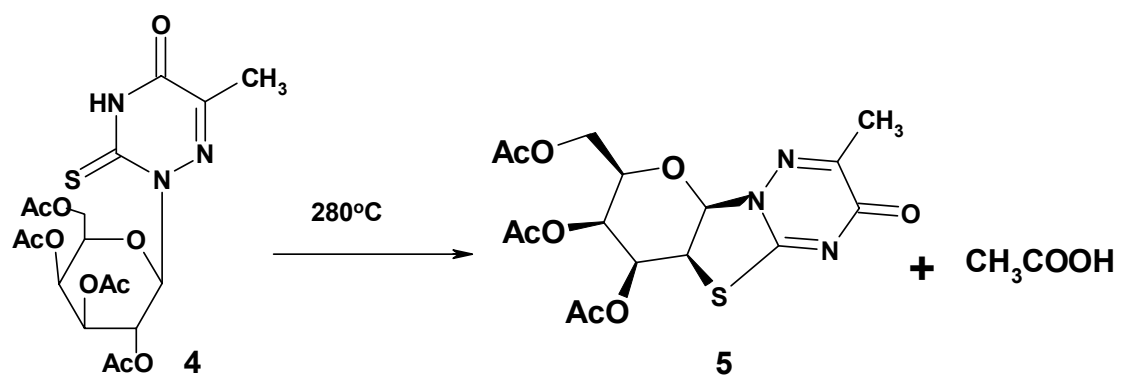

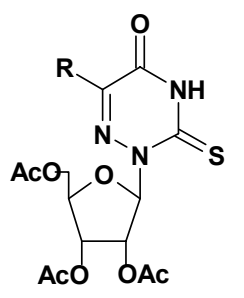

6

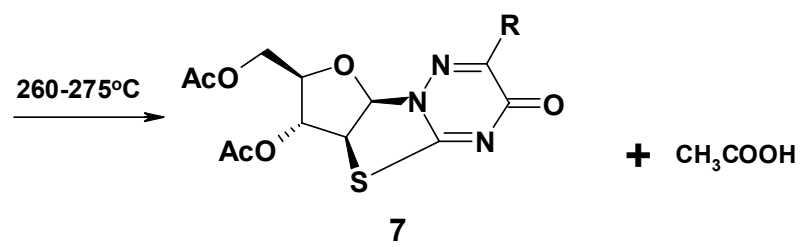

\section{Scheme 2}

It is believed that the anhydronucleosides are formed via intramolecular $\mathrm{S}_{N} 2$ attack (5-exotet) of the sulfur on to the C-2' of the glycosyl moiety to eliminate the acetate $\left(\mathrm{CH}_{3} \mathrm{COO}^{-}\right)$in the form of acetic acid (Scheme 3). ${ }^{14}$
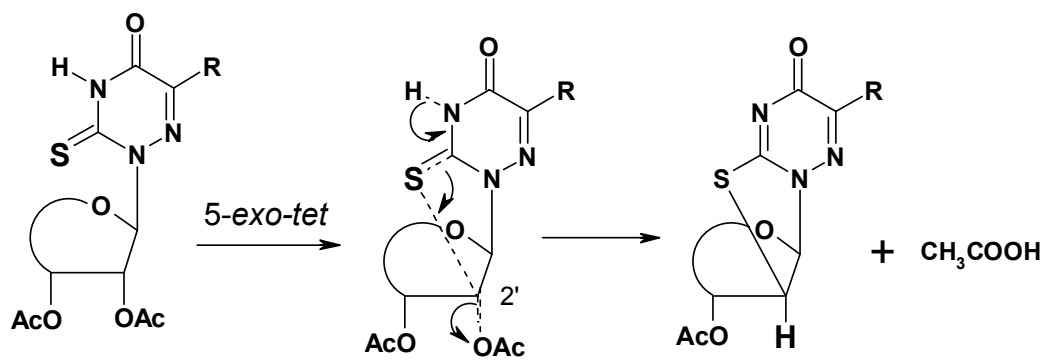

\section{Scheme 3}

\section{Results and Discussion}

In the present work we extended our study to the synthesis of the thio-anhydroglycosyls of 1,2,4triazole derivatives, 12. Scheme 4 illustrates our synthetic strategy towards the starting precursor 2-glycosyl-1,2,4-triazole derivatives, 11. Thus, glycosylation of 4-arylideneamino-1,2,4-triazole2H-3-thiones, 8, with acetobromoglucose in the presence of $\mathrm{NaH}$ in acetonitrile gave a mixture of the corresponding $\mathrm{N}$ - and S-glycosyl derivatives 9 and 10, respectively which were separated by fractional crystallization. ${ }^{12}$ Pyrolysis of either of $\mathbf{9}$ and/or $\mathbf{1 0}$ at $200{ }^{\circ} \mathrm{C}$ led to the elimination 
of benzonitrile derivatives and yielded the same 2-glycosyl-4- $H$-1,2,4-triazole- $2 H$-thione derivatives, 11. When the obtained mixture of $\mathbf{9}$ and $\mathbf{1 0}$ was heated, also, the same products $\mathbf{1 1}$ were obtained at $200{ }^{\circ} \mathrm{C}$. Thus, a separation step is not required.

After several trials the synthesis of anhydrothioglycosyls of 1,2,4-triazoles 12 was achieved upon pyrolyzing the immediate precursors $\mathbf{1 1}$ and the yield of the latter was optimized upon heating at $270-280{ }^{\circ} \mathrm{C}$. The formation of the products and the disappearance of the starting materials were confirmed by LCMS. Direct conversion of each of $\mathbf{9}$ and $\mathbf{1 0}$ to the corresponding anhydro derivatives $\mathbf{1 2}$ has also been achieved by heating at $280{ }^{\circ} \mathrm{C}$ in the static pyrolyzer.

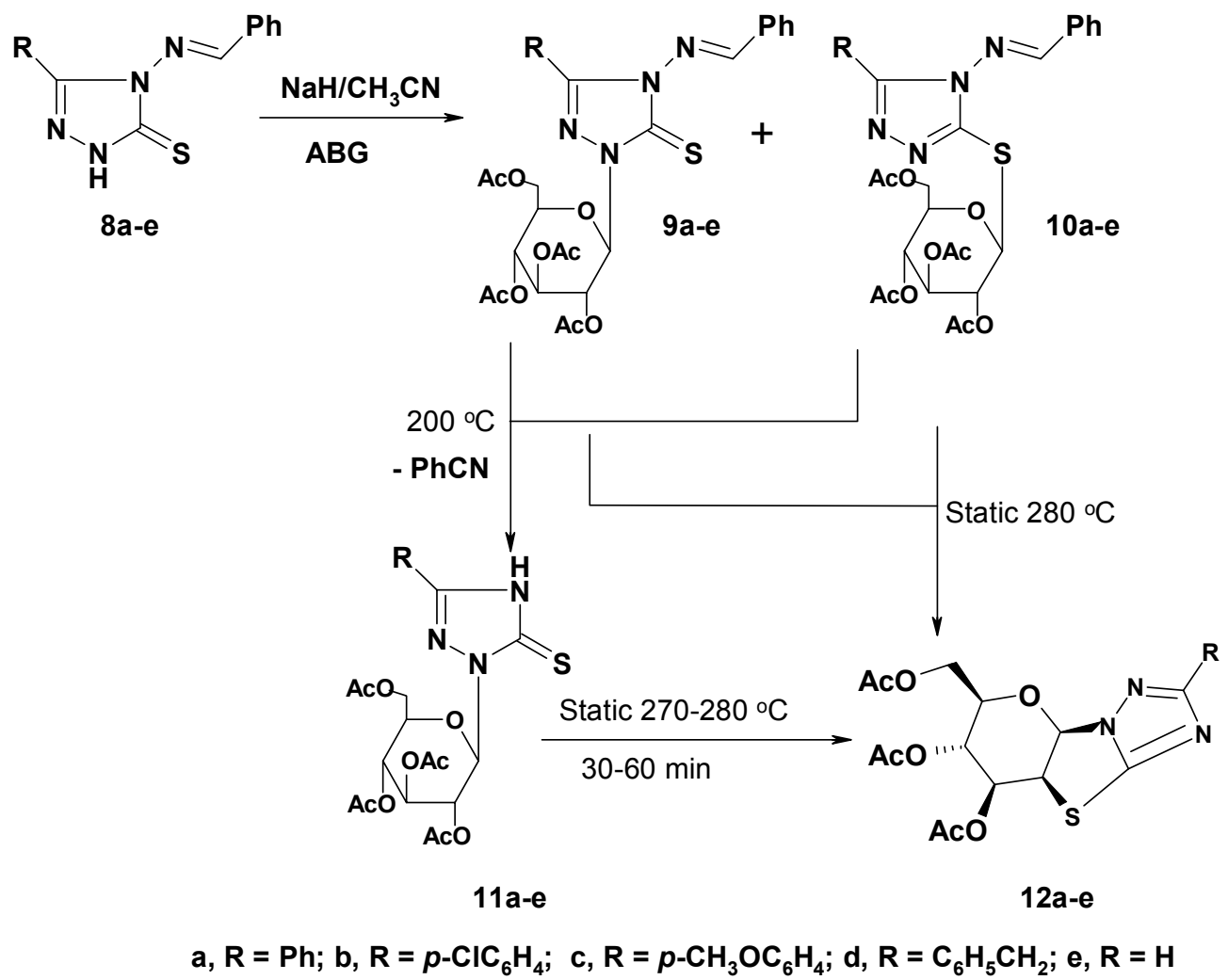

\section{Scheme 4}

The structure of the glycosyl derivatives 9-11 and their corresponding anhydro derivatives, 12, was established based on their MS, LCMS, and NMR data. Table 1 shows the ${ }^{1} \mathrm{H}-\mathrm{NMR}$ chemical shifts of the anomeric $\mathrm{H}$ of the $\mathrm{N}$-glycosides, 9, 11, the S-glycosides 10, and the anhydro derivatives, 12, obtained in the present study. The structures of the anhydro- mannosyl derivatives 12 were determined from the ${ }^{1} \mathrm{H}$ - NMR data of the anomeric hydrogen. Thus, whereas the anomeric protons of $\beta$ - $N$-glycosides 9, 11 appear at high frequencies, $\delta=6.01-6.34$ with large $J$ values $(J=9.0-9.6 \mathrm{~Hz})$, the anhydro products 12 have anomeric proton signals that are shifted to lower frequencies, $\delta=5.88-5.91$, with small coupling constants $(J=3.6 \mathrm{~Hz})$ (Table 1, Experimental Section). This shielding is attributed to the removal of the acetoxy group with its 
inductive and anisotropic effect. The change of the coupling constant depends on the dihedral angle, which is large (1,2-axial, axial) in 9-11, and small (1,2-axial, equatorial) in $\mathbf{1 2}$.

Table 1. ${ }^{1} \mathrm{H}$ NMR signals of the anomeric $\mathrm{H}$ of compounds 9-12

\begin{tabular}{|c|c|c|c|}
\hline Structure & Compound no.* & ${ }^{1} \mathrm{H} \mathrm{NM}$ & $(\mathrm{J} \mathrm{Hz})$ anomeric $\mathrm{H}$ \\
\hline & $\begin{array}{l}9 a \\
9 b \\
9 c \\
9 d \\
9 e\end{array}$ & $\begin{array}{l}\mathrm{C}_{6} \mathrm{H}_{5} \\
p-\mathrm{ClC}_{6} \mathrm{H}_{4} \\
p-\mathrm{CH}_{3} \mathrm{OC}_{6} \mathrm{H}_{4} \\
\mathrm{C}_{6} \mathrm{H}_{5} \mathrm{CH}_{2} \\
\mathrm{H}\end{array}$ & $\begin{array}{l}6.34(9.0)^{12} \\
6.32(9.2) \\
6.31(9.6) \\
6.21(9.6) \\
6.26(9.0)^{12}\end{array}$ \\
\hline & $\begin{array}{l}10 a \\
10 b \\
10 c\end{array}$ & $\begin{array}{l}\mathrm{C}_{6} \mathrm{H}_{5} \\
p-\mathrm{ClC}_{6} \mathrm{H}_{4} \\
p-\mathrm{CH}_{3} \mathrm{OC}_{6} \mathrm{H}_{4}\end{array}$ & $\begin{array}{l}5.49(10.0)^{12} \\
5.47(10.4) \\
5.45(10.4)\end{array}$ \\
\hline & $\begin{array}{l}11 \mathrm{a} \\
11 \mathrm{~b} \\
11 \mathrm{c} \\
11 \mathrm{~d} \\
11 \mathrm{e}\end{array}$ & $\begin{array}{l}\mathrm{C}_{6} \mathrm{H}_{5} \\
p-\mathrm{ClC}_{6} \mathrm{H}_{4} \\
p-\mathrm{CH}_{3} \mathrm{OC}_{6} \mathrm{H}_{4} \\
\mathrm{C}_{6} \mathrm{H}_{5} \mathrm{CH}_{2} \\
\mathrm{H}\end{array}$ & $\begin{array}{l}6.13(9.4)^{12} \\
6.13(9.2) \\
6.13(9.6) \\
6.01(9.2) \\
6.07(9.0)^{12}\end{array}$ \\
\hline & $\begin{array}{l}12 \mathrm{a} \\
12 \mathrm{~b} \\
12 \mathrm{c} \\
12 \mathrm{~d} \\
12 \mathrm{e}\end{array}$ & $\begin{array}{l}\mathrm{C}_{6} \mathrm{H}_{5} \\
p-\mathrm{ClC}_{6} \mathrm{H}_{4} \\
p-\mathrm{CH}_{3} \mathrm{OC}_{6} \mathrm{H}_{4} \\
\mathrm{C}_{6} \mathrm{H}_{5} \mathrm{CH}_{2} \\
\mathrm{H}\end{array}$ & $\begin{array}{l}5.90(3.6) \\
5.91(3.6) \\
5.91(3.6) \\
5.80(3.6) \\
5.88(3.6)\end{array}$ \\
\hline
\end{tabular}

*Compounds 10d,e could not be obtained in pure state to report their accurate data.

Assignments of the ring- protons and carbons of compounds 12a are shown in Figure 1. They are based on $H, H$-COSY, HMQC and HMBC experiments. 

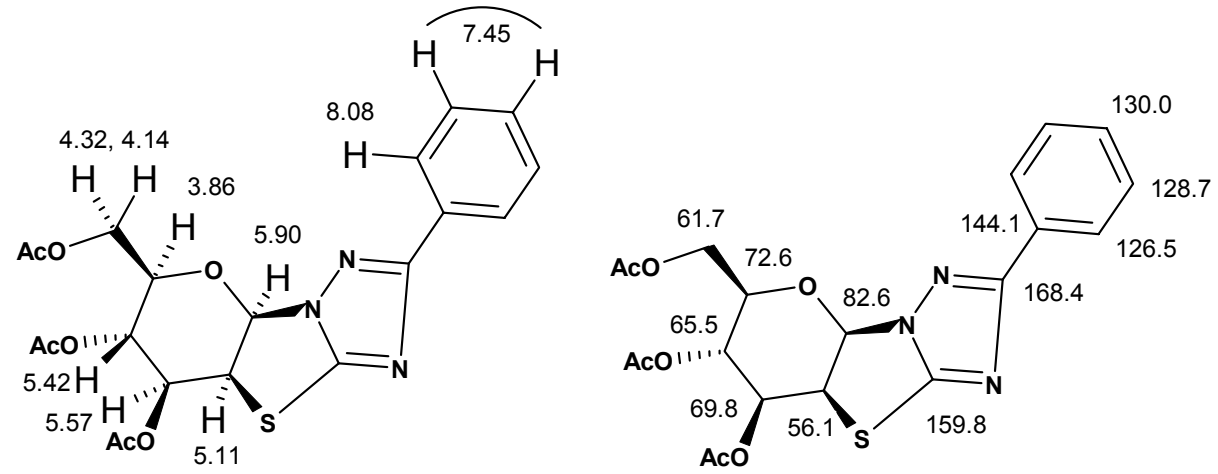

Figure 1. ${ }^{1} \mathrm{H}$ - and ${ }^{13} \mathrm{C}$ - NMR assignments for 12a. The HMBC correlation showed the following $\mathrm{H}-\mathrm{C}$ cross peaks: $o$-phenyl-H at $\delta 8.08$ correlates with triazole $\mathrm{C}-5$ (at $\delta 168.4$ ), and the anomeric $\mathrm{H}$ at $\delta 5.90$ correlates with triazole $\mathrm{C}-3$ (at $\delta$ 159.8). Other protons and carbons are readily deduced from the cross-direct 2-D H,H-COSY, HMQC correlations.

\section{Conclusions}

The present study successfully extends our recently reported pyrolytic synthesis of anhydrothioglycosyls to the 1,2,4-triazole derivatives. The results obtained are promising and encourage us to continue further work to convert other glycosyl derivatives into their anhydroglycosyl derivatives, which will be valuable starting materials for the synthesis of new potential biologically active glycosyl derivatives.

\section{Experimental Section}

General Procedures. All melting points are uncorrected. IR spectra were recorded in $\mathrm{KBr}$ disks on a Perkin-Elmer System 2000 FT-IR spectrophotometer. ${ }^{1} \mathrm{H}$ - and ${ }^{13} \mathrm{C}$ - NMR spectra were recorded on Bruker DPX 400, $400 \mathrm{MHz}$, Avance ${ }^{\mathrm{II}}$ 600, $600 \mathrm{MHz}$, super-conducting NMR spectrometers. Mass spectra were measured on VG Autospec-Q (high resolution, high performance, tri-sector GC/MS/MS) and with LCMS using an Agilent 1100 series LC/MSD with an API-ES/APCI ionization mode. Microanalyses were performed on a LECO CH NS-932 Elemental Analyzer. Separation of reaction products was performed using a preparative HPLC, WATERS PREP 4000 series with PDA detector WATERS 2996. Compounds 9a,e, 10a, 11a,d,e were prepared as reported. ${ }^{12}$ 


\section{Synthesis of glycosyl derivatives 9 and 10 . General procedure}

To a mixture of each of 8a-e $(10 \mathrm{mmol})$, in dry acetonitrile $(50 \mathrm{~mL})$, and $\mathrm{NaH}(12 \mathrm{mmol})$ after stirring together under nitrogen for $30 \mathrm{~min}$ was added acetobromoglucose (12 mmol). The reaction mixture was then stirred overnight at RT. The solvent was then removed in vacuo and ice water $(50 \mathrm{~mL})$ was added. The product was collected and washed several times with water. Upon crystallization from ethanol compounds 9 crystallized out first, and upon concentration of the mother liquor compounds $\mathbf{1 0}$ crystallized out. The latter were recrystallized from ethanol to give pure 10.

4-Benzylideneamino-5-phenyl-2-(2,3,4,6-tetra- $O$-acetyl- $\beta$-D- $N$-glucopyranosyl)-1,2,4triazole-3(4H)-thione (9a). Colorless crystals from ethanol, yield 50\%, mp 200-202 ${ }^{\circ} \mathrm{C}$ (lit. ${ }^{12} \mathrm{mp}$ 202-204 $\left.{ }^{\circ} \mathrm{C}\right)$. MS: $m / z=610\left(\mathrm{M}^{+}, 85 \%\right)$. LCMS: $m / z=611(M+1)$.

\section{4-Benzylideneamino-5-p-chlorophenyl-2-(2,3,4,6-tetra- $O$-acetyl- $\beta$-D- $N$-glucopyranosyl)-}

1,2,4-triazole-3(4H)-thione (9b). Colorless crystals from ethanol, yield 60\%, mp 202-203 ${ }^{\circ} \mathrm{C}$ MS: $m / z=644\left(\mathrm{M}^{+}\right), 646(\mathrm{M}+2) . \mathrm{IR}: 3082,1748,1604,1490,1427,1366,1320,1248,1224$, 1094, 1065, 1036, 1014, $759 \mathrm{~cm}^{-1} .{ }^{1} \mathrm{H}$ NMR $\left(\mathrm{CDCl}_{3}\right) \delta 10.10(\mathrm{~s}, 1 \mathrm{H}), 7.95-7.87(\mathrm{~m}, 4 \mathrm{H}), 7.60(\mathrm{~m}$, $1 \mathrm{H}), 7.53(\mathrm{~d}, 2 \mathrm{H}, J 7.6), 7.51-7.46(\mathrm{~m}, 2 \mathrm{H}), 6.32(\mathrm{~d}, 1 \mathrm{H}, J 9.2), 5.96(\mathrm{t}, 1 \mathrm{H}, J 9.4), 5.47(\mathrm{t}, 1 \mathrm{H}, J$ 9.4), 5.31 (t, 1H, $J$ 9.8), 4.34 (dd, 1H, $J 12.4,4.8), 4.21$ (dd, 1H, $J 12.4,2.0), 4.06$ (ddd, 1H, $J$ 10.0, 4.8, 2.0), 2.11, 2.10, 2.07, $1.97\left(4 \mathrm{~s}, 4 \mathrm{xCH}_{3}\right) .{ }^{13} \mathrm{C} \mathrm{NMR}\left(\mathrm{CDCl}_{3}\right) \delta 170.7,170.2,169.5$, 169.0, 165.0, 164.5, 148.4, 137.5, 133.0, 132.0, 130.3, 129.2, 129.0 (2C), 123.4, 82.1, 74.6, 73.7, 68.9, 67.7, 61.7, 20.8, 20.7, 20.65 (2C). Anal. Calc. for $\mathrm{C}_{29} \mathrm{H}_{29} \mathrm{ClN}_{4} \mathrm{O}_{9} \mathrm{~S}$ (645.1): C 54.0; $\mathrm{H} 4.53$; N 8.69; S 4.97. Found: C 53.74; H 4.80; N 8.67; S 5.20\%.

4-Benzylidene-amino-5-p-methoxyphenyl-2-(2,3,4,6-tetra- $O$-acetyl- $\beta$-D- $N$-glucopyranosyl)1,2,4-triazole-3(4H)-thione (9c). Colorless crystals from ethanol, yield 40\%, mp $206{ }^{\circ} \mathrm{C} . \mathrm{MS}$ : $m / z=640\left(\mathrm{M}^{+}\right) . \mathrm{IR}: 3011,2966,2943,1750,1609,1505,1431,1368,1257,1243,1229,1182$, 1103, 1067, $1037 \mathrm{~cm}^{-1} .{ }^{1} \mathrm{H}$ NMR $\left(\mathrm{CDCl}_{3}\right) \delta 10.04(\mathrm{~s}, 1 \mathrm{H}), 7.89(\mathrm{~m}, 4 \mathrm{H}), 7.54(\mathrm{~m}, 3 \mathrm{H}), 6.99$ (d, $2 \mathrm{H}, J 8.8), 6.31$ (d, 1H, J 9.6), 5.99 (t, 1H, J 9.2), 5.46 (t, 1H, J 9.4), 5.31 (t, 1H, J 9.8), 4.34 (dd, $1 \mathrm{H}, J 12.6,4.6), 4.21(\mathrm{~d}, 1 \mathrm{H}, J 12.4), 4.05(\mathrm{~m}, 1 \mathrm{H}), 3.89(\mathrm{~s}, 3 \mathrm{H}), 2.11,2.09,2.07,1.97(4 \mathrm{~s}$, $\left.4 \mathrm{xCH}_{3}\right) .{ }^{13} \mathrm{C} \mathrm{NMR}\left(\mathrm{CDCl}_{3}\right): \delta 170.7,170.2,169.4,168.9,164.8,164.6,161.8,149.1,132.8$, 132.2, 130.6, 129.1, 129.0, 117.3, 114.1, 82.2, 74.5, 73.8, 68.9, 67.8, 61.7, 55.4, 20.8, 20.7, 20.6 (2C). Anal. Calc. for $\mathrm{C}_{30} \mathrm{H}_{32} \mathrm{~N}_{4} \mathrm{O}_{10} \mathrm{~S}$ (640.7): C 56.24; H 5.03; N 8.74; S 5.00. Found: C 56.05; H 4.97; N 8.79; S 5.31\%.

5-Benzyl-4-benzylidene-amino-2-(2,3,4,6-tetra-O-acetyl- $\beta$-D- $N$-glucopyranosyl)-1,2,4triazole-3(4H)-thione (9d). Colorless crystals from ethanol, yield 55\%, mp. 143-144 ${ }^{\circ} \mathrm{C}$. MS: $m / z=624\left(\mathrm{M}^{+}\right)$. IR: 3059, 3033, 2917, 1754, 1427, 1367, 1316, 1222, 1059, 1036, 909, 730 $\mathrm{cm}^{-1}$. ${ }^{1} \mathrm{H}-\mathrm{NMR}\left(\mathrm{CDCl}_{3}\right): \delta 10.32(\mathrm{~s}, 1 \mathrm{H}), 7.83(\mathrm{dd}, 2 \mathrm{H}, J$ 8.4, 1.2), $7.56(\mathrm{t}, 1 \mathrm{H}, J$ 7.2), $7.50(\mathrm{t}, 2 \mathrm{H}, J$ 7.4), 7.33-7.29 (m, 5H), $6.21(\mathrm{~d}, 1 \mathrm{H}, J 9.6), 5.89$ (t, 1H, $J 9.4), 5.43$ (t, 1H, J 9.6), $5.30(\mathrm{t}, 1 \mathrm{H}, J$ 9.6), 4.32 (dd, 1H, J 12.8, 4.8), 4.20 (m, 3H), 4.02 (ddd, 1H, J 10.4, 4.8, 2.4), 2.12, 2.09, 2.06, $1.89\left(4 \mathrm{~s}, 4 \mathrm{xCH}_{3}\right) .{ }^{13} \mathrm{C} \mathrm{NMR}\left(\mathrm{CDCl}_{3}\right): \delta 170.8,170.3,169.4,168.9,164.1,161.3,150.9,134.3$, 132.6, 132.4, 129.04, 129.01, 128.8, 128.7, 127.3, 81.5, 74.5, 73.8, 68.9, 67.8, 61.7, 31.3, 20.8, 20.7 (2C), 20.6. Anal. Calc. for $\mathrm{C}_{30} \mathrm{H}_{32} \mathrm{~N}_{4} \mathrm{O}_{9} \mathrm{~S}$ (624.7): C 57.68; H 5.16; N 8.97 ; S 5.13. Found: 
C, 58.12; H, 5.11; N, 9.09; S, 5.29\%.

4-Benzylidene-amino-5-phenyl-3-(2,3,4,6-tetra- $O$-acetyl- $\beta$-D- $N$-glucopyranosylsulfanyl)-4H1,2,4-triazole (10a). Colorless crystals from ethanol, yield 18\%, mp 192-93 ${ }^{\circ} \mathrm{C}$ (lit. ${ }^{12} \mathrm{mp} .193{ }^{\circ} \mathrm{C}$ ). MS: $m / z=644.5$. LCMS: $m / z=611(M+1)$.

4-Benzylidene-amino-5-p-chlorophenyl-3-(2,3,4,6-tetra- $O$-acetyl- $\beta$-D- $N$-glucopyranosyl-

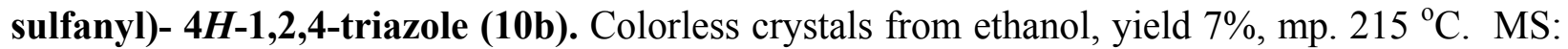
$m / z=644\left(M^{+}\right), 646(M+2)$. IR : 3265, 2943, 1753, 1432, 1376, 1229, 1092, 1061, 1045. ${ }^{1} \mathrm{H}-$ NMR $\left(\mathrm{CDCl}_{3}\right) \delta 8.68(\mathrm{~s}, 1 \mathrm{H}), 7.95(\mathrm{dd}, 2 \mathrm{H}, J$ 8.6), $7.87(\mathrm{dd}, 2 \mathrm{H}, J$ 8.4, 1.2), $7.65(\mathrm{t}, 1 \mathrm{H}, J$ 7.6), 7.56 (t, 2H, $J$ 7.6), 7.46 (d, 2H, $J$ 8.6), 5.47 (d, 1H, $J 10.4), 5.29$ (t, 1H, $J 9.2), 5.17$ (t, 1H, $J 9.6)$, $5.13(\mathrm{t}, 1 \mathrm{H}, J$ 9.6), 4.28 (dd, 1H, J 12.8, 4.8), 4.03 (dd, 1H, J 12.4, 1.6), 3.78 (m, 1H), 2.08, 2.05, 2.03, $1.97\left(4 \mathrm{~s}, 4 \mathrm{xCH}_{3}\right) .{ }^{13} \mathrm{C}-\mathrm{NMR}\left(\mathrm{CDCl}_{3}\right) \delta 170.5,170.0,169.7,169.5,167.4,151.7,143.6$, 136.5, 133.6, 131.3, 129.5, 129.3, 129.1, 124.8, 124.76, 85.4, 76.3, 73.6, 70.0, 67.7, 61.6, 20.9, 20.85, 20.8 (2C). Anal. Calc. for $\mathrm{C}_{29} \mathrm{H}_{29} \mathrm{ClN}_{4} \mathrm{O}_{9} \mathrm{~S}$ (645.1): C 54.0; H 4.53; N 8.69; S 4.97. Found: C 53. 57; H 4.81; N 8.71; S 4.95\%.

4-Benzylidene-amino-5-p-methoxyphenyl-3-(2,3,4,6-tetra- $O$-acetyl- $\beta$-D- $N$-glucopyranosylsulfanyl)-4H-1,2,4-triazole (10c). Colorless crystals from ethanol, yield 10\%, mp. 111-112 ${ }^{\circ} \mathrm{C}$. MS: $m / z=640\left(\mathrm{M}^{+}\right)$. IR : 3014, 2941, 2900, 1752, 1610, 1469, 1437, 1367, 1258, 1228, 1213 , 1181, 1089, 1045, $1031 \mathrm{~cm}^{-1} .{ }^{1} \mathrm{H}-\mathrm{NMR}\left(\mathrm{CDCl}_{3}\right) \delta 8.66(\mathrm{~s}, 1 \mathrm{H}), 7.92(\mathrm{~d}, 2 \mathrm{H}, J 8.8), 7.87(\mathrm{~d}, 2 \mathrm{H}$, $J 7.2), 7.63$ (t, 1H, J 7.2), 7.54 (t, 2H, J 7.2 ), $6.98(\mathrm{~d}, 2 \mathrm{H}, J 8.8), 5.45(\mathrm{~d}, 1 \mathrm{H}, J 10.4), 5.29(\mathrm{t}, 1 \mathrm{H}$, $J 9.6), 5.16$ (t, 1H, J 9.6), 5.12 (t, 1H, J 9.6), 4.27 (dd, 1H, J 12.8, 4.8), 4.03 (dd, 1H, $J 12.8,1.0)$, $3.87(\mathrm{~s}, 3 \mathrm{H}), 3.77(\mathrm{ddd}, 1 \mathrm{H}, J 10.2,4.8,1.0), 2.09,2.04,2.03,1.97\left(4 \mathrm{~s}, 4-\mathrm{CH}_{3}\right) .{ }^{13} \mathrm{C}-\mathrm{NMR}$ $\left(\mathrm{CDCl}_{3}\right): \delta 170.5,170.0,169.7,169.5,167.0,161.1,152.4,143.1,133.4,131.6,129.9,129.3$ (2C), 118.8, 114.2, 85.4, 76.2, 73.7, 70.1, 67.8, 61.6, 55.4, 20.7, 20.6, 20.6 (2C). Anal. Calc. for $\mathrm{C}_{30} \mathrm{H}_{32} \mathrm{~N}_{4} \mathrm{O}_{10} \mathrm{~S}$ (640.7): C 56.24; $\mathrm{H} 5.03 ; \mathrm{N} 8.74 ; \mathrm{S} 5.00$. Found: C 55.85; H 5.09; N 8.6; S $5.33 \%$.

\section{2-(2,3,4,6-Tetra- $O$-acetyl- $\beta$-D- $N$-glucopyranosyl)-1,2,4-triazole-3-(4H)-thiones (11a-e).} General procedures

(A) Each of the compounds 9a-e and 10a-c $(0.2 \mathrm{~g})$ was introduced in the reaction tube, cooled in liquid nitrogen, sealed under vacuum, and placed in the pyrolyzer at $200{ }^{\circ} \mathrm{C}$ (static pyrolyzer) for $45 \mathrm{~min}$. The contents of the tube were dissolved and crystallized from ethanol to give white crystals of the compounds $11 \mathbf{a}-\mathbf{c}$.

(B) The apparatus used was similar to that described in our recent publications. ${ }^{15 a-c}$ Each mixture (obtained from 8) of compounds 9, $10(1 \mathrm{mmol})$ was volatilized from a tube heated at $200{ }^{\circ} \mathrm{C}$ for $45 \mathrm{~min}$ in a Büchi Kügelrohr oven through a $30 \times 2.5 \mathrm{~cm}$ horizontal fused quartz tube. This was heated externally by a Carbolite Eurotherm furnace MTF-12/38A at a temperature of $350{ }^{\circ} \mathrm{C}$, the temperature being monitored by a $\mathrm{Pt} / \mathrm{Pt}-13 \% \mathrm{Rh}$ thermocouple situated at the centre of the furnace. The product was collected in the sublimation tube while benzonitrile was collected in a U-shaped trap cooled in liquid nitrogen. The whole system was maintained at a pressure of $10^{-2}$ Torr by an Edwards model E2M5 high capacity rotary oil pump, the pressure being measured by 
a Pirani gauge situated between the cold trap and the pump. The product was dissolved and crystallized from ethanol to give white crystals of compound $\mathbf{1 1 b}$.

5-Phenyl-2-(2,3,4,6-tetra- $O$-acetyl- $\beta$-D-N-glucopyranosyl)-1,2,4-triazole-3(4H)-thione (11a). Prepared using General Procedure A. Colorless crystals from ethanol, yield 78\% (from 9a), 47\% (from 10a), mp 228-30 ${ }^{\circ} \mathrm{C}$ (lit. ${ }^{12} \mathrm{mp} .228{ }^{\circ} \mathrm{C}$ ). MS: $m / z=507\left(\mathrm{M}^{+}, 85 \%\right)$ LCMS: $m / z=508$ $(\mathrm{M}+1)$.

5-p-Chlorophenyl-2-(2,3,4,6-tetra- $O$-acetyl- $\beta$-D- $N$-glucopyranosyl)-1,2,4-triazole-3(4H)thione (11b). Colorless crystals from ethanol, yield 80\% (from 9b) and 75\% (from 10b) using procedure A, 75\% (from a mixture of $\mathbf{9 b}$, and $\mathbf{1 0 b}$ ) using procedure B, mp. $240{ }^{\circ} \mathrm{C} . \mathrm{MS}: \mathrm{m} / z=$ $541\left(\mathrm{M}^{+}\right), 543(\mathrm{M}+2)$. IR: 2920, 2852, 1752, 1612, 1494, 1471, 1434, 1375, 1369, 1240, 1226, 1094, 1045, 837 $\mathrm{cm}^{-1} .{ }^{1} \mathrm{H}$ NMR $\left(\mathrm{CDCl}_{3}\right) \delta 11.97(\mathrm{~s}, 1 \mathrm{H}, \mathrm{NH}), 7.79(\mathrm{~d}, 2 \mathrm{H}, J 8.4), 7.51(\mathrm{~d}, 2 \mathrm{H}, J$ 8.4), 6.13 (d, 1H, J 9.2), 5.84 (t, 1H, J 9.4), 5.46 (t, 1H, J9.4), 5.31 (t, 1H, $J$ 9.8), 4.35 (dd, $1 \mathrm{H}, J$ 12.4, 5.2), $4.20(\mathrm{dd}, 1 \mathrm{H}, J 12.4,2.0), 4.05(\mathrm{ddd}, 1 \mathrm{H}, J 10.0,5.2,2.0), 2.10(\mathrm{~s}, 6 \mathrm{H}), 2.07$ (s, 3H), $1.93(\mathrm{~s}, 3 \mathrm{H}) .{ }^{13} \mathrm{C} \mathrm{NMR}\left(\mathrm{CDCl}_{3}\right): \delta 170.7,170.2,169.4,169.0,168.9,152.8,138.1,129.7,127.6$, 122.7, 82.0, 74.6, 73.5, 69.5, 67.8, 61.7, 20.7, $20.6(2 \mathrm{C}), 20.55$. Anal. Calc. for $\mathrm{C}_{22} \mathrm{H}_{24} \mathrm{~N}_{3} \mathrm{O}_{9} \mathrm{SCl}$ (542.0): C 48.76; H 4.46; N 7.75 ; S 5.92. Found: C 48.45; H 4.51; N 7.70; S 6.20\%.

\section{5-p-Methoxyphenyl-2-(2,3,4,6-tetra- $O$-acetyl- $\beta$-D- $N$-glucopyranosyl)-1,2,4-triazole-3(4H)-}

thione (11c). Prepared using general procedure A. Colorless crystals from ethanol, yield 85\% (from 9c), 47\% (from 10c), mp. 230-231 ${ }^{\circ} \mathrm{C} . \mathrm{MS}: m / z=537\left(\mathrm{M}^{+}\right.$). IR : 3017, 2958, 2919, 2849, 1754, 1616, 1510, 1470, 1443, 1368, 1247, 1229, 1180, 1098, 1060, 1042, 911, 733. ${ }^{1} \mathrm{H}$ NMR $\left(\mathrm{CDCl}_{3}\right) \delta 12.40(\mathrm{~s}, 1 \mathrm{H}, \mathrm{NH}), 7.81(\mathrm{~d}, 2 \mathrm{H}, J 8.8), 7.02(\mathrm{~d}, 2 \mathrm{H}, J 8.4), 6.13(\mathrm{~d}, 1 \mathrm{H}, J 9.6), 5.86(\mathrm{t}$, $1 \mathrm{H}, J$ 9.4), 5.46 (t, 1H, J 9.4), 5.31 (t, 1H, J 9.8), 4.35 (dd, 1H, $J 12.4,4.6), 4.20$ (d, 1H, $J 12.4)$, $4.05(\mathrm{~m}, 1 \mathrm{H}), 3.90(\mathrm{~s}, 3 \mathrm{H}), 2.10(\mathrm{~s}, 6 \mathrm{H}), 2.06(\mathrm{~s}, 3 \mathrm{H}), 1.91(\mathrm{~s}, 3 \mathrm{H}) .{ }^{13} \mathrm{C} \mathrm{NMR}\left(\mathrm{CDCl}_{3}\right): \delta 170.7$, 170.2, 169.4, 168.8, 168.3, 162.3, 149.9, 128.1, 116.7, 114.7, 82.0, 74.6, 73.6, 69.4, 67.8, 61.7, 55.5, 20.8, 20.63 (2C), 20.56. Anal. Calc. for $\mathrm{C}_{23} \mathrm{H}_{27} \mathrm{~N}_{3} \mathrm{O}_{10} \mathrm{~S}$ (537.6): C 51.39; H 5.03; N 7.82; $\mathrm{S}$ 5.95. Found: C 51.10; H 5.27; N 7.53; S 5.97\%.

5-Benzyl-2-(2,3,4,6-tetra-O-acetyl- $\beta$-D- $N$-glucopyranosyl)-1,2,4-triazole-3(4H)-thione (11d). Prepared using general procedure A. Colorless crystals from ethanol, yield 92\% (from 9d), mp 141-142 ${ }^{\circ} \mathrm{C}$ LCMS: $m / z=522(\mathrm{M}+1)$. IR: 3089, 2991, 1753, 1475, 1367, 1237, 1220, 1095, 1062, 1033, 1009, 756, 728. ${ }^{1} \mathrm{H}$ NMR $\left(\mathrm{CDCl}_{3}\right) \delta 11.62(\mathrm{~s}, 1 \mathrm{H}, \mathrm{NH}), 7.37-7.23(\mathrm{~m}, 5 \mathrm{H}), 6.01(\mathrm{~d}$, $1 \mathrm{H}, J$ 9.2), 5.73 (t, 1H, J 9.4), 5.42 (t, 1H, J 9.4), 5.29 (t, 1H, J 9.8), 4.33 (dd, 1H, J 12.8, 4.8), $4.19(\mathrm{~d}, 1 \mathrm{H}, J 12.8), 4.06(\mathrm{~d}, 1 \mathrm{H}, J 16.0), 4.03(\mathrm{~m}, 1 \mathrm{H}), 3.90(\mathrm{~d}, 1 \mathrm{H}, J 16.0), 2.11,2.09,2.05$, $1.88\left(4 \mathrm{~s}, 4 \mathrm{xCH}_{3}\right) .{ }^{13} \mathrm{C} \mathrm{NMR}\left(\mathrm{CDCl}_{3}\right): \delta 170.8,170.2,169.4,168.9,168.7,150.6,133.5,129.3$, $128.9,128.0,81.8,74.6,73.4,69.5,67.8,61.7,32.3,20.8,20.6$ (2C), 20.5. Anal. Calc. for $\mathrm{C}_{23} \mathrm{H}_{27} \mathrm{~N}_{3} \mathrm{O}_{9} \mathrm{~S}$ (521.6): C 52.97; H 5.22; N 8.06; S 6.15. Found: C 53.27; H 4.97; N 8.21; S $5.95 \%$.

3,2'-Anhydro-2-(3,4,6-tri-O-acetyl- $\beta$-D-mannopyranosyl)-2H-1,2,4-triazol-3-thiols (12a-e). General procedure

Each of the compounds 9a,e, 10a,e and/or 11a-e $(0.2 \mathrm{~g})$ was separately introduced into the reaction tube, cooled in liquid nitrogen, sealed under vacuum, and placed in the static pyrolyzer 
at $270-280{ }^{\circ} \mathrm{C}$ for $30-60 \mathrm{~min}\left(11 \mathrm{a}, 280{ }^{\circ} \mathrm{C}, 30 \mathrm{~min} ; \mathbf{1 1 b}, 270{ }^{\circ} \mathrm{C}, 60 \mathrm{~min} ; 11 \mathrm{c}, 270{ }^{\circ} \mathrm{C}, 30 \mathrm{~min}\right.$; 11d,e, $270{ }^{\circ} \mathrm{C}, 30 \mathrm{~min}$.). After cooling, the content of the tube was dissolved in $\mathrm{CH}_{2} \mathrm{Cl}_{2}$ and stirred with saturated $\mathrm{NaHCO}_{3}$ solution $\left(5 \mathrm{~g}\right.$ in $\left.10 \mathrm{~mL} \mathrm{H}_{2} \mathrm{O}\right)$ for $2 \mathrm{~h}$. The organic phase was then separated, dried over anhydrous sodium sulfate and evaporated on a Rotavap under reduced pressure to give the corresponding products 12a-e, respectively.

3,2'-Anhydro-5-phenyl-2-(3,4,6-tri- $\mathrm{O}$-acetyl- $\beta$-D-mannopyranosyl)-2H-1,2,4-triazol-3-thiol (12a). Colorless crystals from $\mathrm{CHCl}_{3} /$ petroleum, yield $46 \%$ (from 9a), $40 \%$ (from 10a), $38 \%$ (from 11a), mp 172-74 ${ }^{\circ} \mathrm{C}$. MS: $m / z=447\left(\mathrm{M}^{+}, 65 \%\right), 272$ (45\%), 177 (100\%). LCMS: $m / z=$ $448(\mathrm{M}+1)$. IR: 2928, 1752, 1635, 1484, 1445, 1369, 1227, 1062, 910, 785, 726. ${ }^{1} \mathrm{H}$ NMR $\left(\mathrm{CDCl}_{3}\right) \delta 8.08(\mathrm{dd}, 2 \mathrm{H}, J 7.8,1.8), 7.45(\mathrm{~m}, 3 \mathrm{H}), 5.90(\mathrm{~d}, 1 \mathrm{H}, J 3.6), 5.57$ (dd, $1 \mathrm{H}, J$ 9.6, 6.0), 5.42 (t, $1 \mathrm{H}, J$ 9.6), 5.11 (dd, $1 \mathrm{H}, J$ 6.0, 3.6), 4.32 (dd, $1 \mathrm{H}, J 12.6,4.8$ ), 4.14 (dd, $1 \mathrm{H}, J 12.6,2.4$ ), 3.86 (ddd, $1 \mathrm{H}, J 12.6,4.8,2.4), 2.19,2.11,2.07\left(3 \mathrm{~s}, 9 \mathrm{H}, 3 \mathrm{xCH}_{3}\right) .{ }^{13} \mathrm{C} \mathrm{NMR}\left(\mathrm{CDCl}_{3}\right) \delta 170.7$, 169.7, 169.3, 168.4, 159.8, 144.1, 130.0, 128.7, 126.5, 82.6, 72.6, 69.8, 65.5, 61.7, 56.1, 20.8, 20.7, 20.6. Anal. Calc. for $\mathrm{C}_{20} \mathrm{H}_{21} \mathrm{~N}_{3} \mathrm{O}_{7} \mathrm{~S}$ (447.39): C, 53.69; H, 4.69; N, 9.39; S, 7.15. Found: C, $53.50 ; \mathrm{H}, 4.60 ; \mathrm{N}, 9.17 ; \mathrm{S}, 7.20 \%$.

3,2'-Anhydro-5-p-chlorophenyl-2-(3,4,6-tri- $O$-acetyl- $\beta$-D-mannopyranosyl)-2H-1,2,4triazol-3-thiol (12b). Yield 38\%, HRMS $=481.0703$ (Calc. for $\mathrm{C}_{20} \mathrm{H}_{20} \mathrm{~N}_{3} \mathrm{O}_{7} \mathrm{SCl}, 481.0705$ ). ${ }^{1} \mathrm{H}$ NMR $\left(\mathrm{CDCl}_{3}\right) \delta 8.02(\mathrm{~d}, 2 \mathrm{H}, J$ 8.8), $7.45(\mathrm{~d}, 2 \mathrm{H}, J 8.8), 5.91(\mathrm{~d}, 1 \mathrm{H}, J 3.6), 5.57$ (dd, $1 \mathrm{H}, J$ 9.6, 6.0), 5.43 (t, $1 \mathrm{H}, J 9.6$ ), 5.12 (dd, $1 \mathrm{H}, J 6.0,3.6$ ), 4.34 (dd, $1 \mathrm{H}, J 12.4,4.8$ ), 4.15 (dd, $1 \mathrm{H}, J 12.4$, 2.4), 3.88 (ddd, $1 \mathrm{H}, J 9.6,4.8,2.4), 2.16,2.10,2.08\left(3 \mathrm{~s}, 9 \mathrm{H}, 3 \mathrm{xCH}_{3}\right)$.

3,2'-Anhydro-5-( $p$-methoxyphenyl)-2-(3,4,6-tri- $O$-acetyl- $\beta$-D-mannopyranosyl)-2H-1,2,4triazol-3-thiol (12c). Yield 40\%, HRMS $=477.1200$ (Calc. for $\mathrm{C}_{21} \mathrm{H}_{23} \mathrm{~N}_{3} \mathrm{O}_{8} \mathrm{~S}, 477.1200$ ). ${ }^{1} \mathrm{H}$ NMR $\left(\mathrm{CDCl}_{3}\right) \delta 8.03(\mathrm{~d}, 2 \mathrm{H}, J 8.8), 6.96(\mathrm{~d}, 2 \mathrm{H}, J 8.8), 5.91(\mathrm{~d}, 1 \mathrm{H}, J 3.6), 5.56(\mathrm{dd}, 1 \mathrm{H}, J$ 9.4, 6.0), 5.42 ( $t, 1 \mathrm{H}, J$ 9.6), 5.11 (dd, $1 \mathrm{H}, J 6.0,3.6$ ), 4.32 (dd, $1 \mathrm{H}, J 12.4,5.2), 4.14$ (dd, $1 \mathrm{H}, J 12.4$, 2.0), $3.85(m, 4 \mathrm{H}), 2.16,2.09,2.07\left(3 \mathrm{~s}, 9 \mathrm{H}, 3 \mathrm{xCH}_{3}\right)$.

3,2'-Anhydro-5-benzyl-2-(3,4,6-tri-O-acetyl-3-D-mannopyranosyl)-2H-1,2,4-triazol-3-thiol (12d). Yield 30\%, HRMS $=461.1250\left(\right.$ Calc. for $\left.\mathrm{C}_{21} \mathrm{H}_{23} \mathrm{~N}_{3} \mathrm{O}_{7} \mathrm{~S}, 461.1251\right) .{ }^{1} \mathrm{H}$ NMR $\left(\mathrm{CDCl}_{3}\right) \delta$ 7.38-7.23 (m, 5H), $5.80(\mathrm{~d}, 1 \mathrm{H}, J 3.6), 5.53(\mathrm{dd}, 1 \mathrm{H}, J 9.4,5.6), 5.37$ (t, $1 \mathrm{H}, J 9.6), 5.02(\mathrm{dd}, 1 \mathrm{H}$, $J$ 5.6, 3.6), $4.28(\mathrm{dd}, 1 \mathrm{H}, J 12.4,5.2), 4.1(\mathrm{~m}, 3 \mathrm{H}), 3.87(\mathrm{~m}, 1 \mathrm{H}), 2.11,2.08,2.05(3 \mathrm{~s}, 9 \mathrm{H}$, $\left.3 \mathrm{xCH}_{3}\right)$.

3,2'-Anhydro-2-(3,4,6-tri- $O$-acetyl- $\beta$-D-mannopyranosyl)-2H-1,2,4-triazol-3-thiol (12e). Yield 36\% (from 9e), 18\% (from 10e), 30\% (from 11e), HRMS = 371.0781 (Calc. for $\left.\mathrm{C}_{14} \mathrm{H}_{17} \mathrm{~N}_{3} \mathrm{O}_{7} \mathrm{~S}, 371.0781\right) .{ }^{1} \mathrm{H}$ NMR $\left(\mathrm{CDCl}_{3}\right) \delta 7.95(\mathrm{~s}, 1 \mathrm{H}), 5.88(\mathrm{~d}, 1 \mathrm{H}, J 3.6), 5.53(\mathrm{dd}, 1 \mathrm{H}, J$ 9.2, 6.0), 5.38 (t, $1 \mathrm{H}, J$ 9.6), 5.10 (dd, $1 \mathrm{H}, J 6.0,3.6), 4.29$ (dd, $1 \mathrm{H}, J 12.4,5.2), 4.18$ (d, $1 \mathrm{H}, J$ $12.4,2.0), 3.85$ (ddd, $1 \mathrm{H}, J 12.4,5.2,2.0), 2.14,2.08,2.07$ (3s, $9 \mathrm{H}, 3 \mathrm{xCH}_{3}$ ). 


\section{Acknowledgements}

The support of Kuwait University received through Research Grant No. SC 08/04 and the facilities of Analab/SAF (Grants No. GS01/01, GS01/03, GS03/01), and the financial support of Ibtehal A. Al-Juwaiser from the College of Graduate Studies are gratefully acknowledged.

\section{References}

1. (a) Mitsuya, H.; Yarchoan, R.; Broder, S. Science 1990, 249, 1533-1544. (b) DeClercq, E. Design of Anti-AIDS Drugs; Elsevier: New York, 1990. (c) Herdewijn, P.; Balzarini, J.; DeClerck, E.; Pauwels, R.; Baba, M.; Broder, S.; Vander-Heghe, H. J. Med. Chem. 1987, 30, 1270-1278. (d) Warshaw, J. A.; Watanabe, K. A.; J. Med. Chem. 1990, 33, 1663-1666. (e) Huang, J.-T.; Chen, L.-C.; Wang, L. Kim, M.-H; Warshaw, J. A.; Armstrong, D.; Zhu, Q.-Y; Chou, T.-C; Watanabe, K. A.; Matulic-Adamic, J.; Su, T.-L; Fox, J. J.; Polsky, B.; Baron, P. A.; Gold, J. W. M.; Hardy, W. D.; Zuckerman, E. J. Med. Chem. 1991, 34, 164046. (f) Rama Rao, A. V.; Gurjar, M. K.; Lalitha, S. V. S. J. Chem. Soc., Chem. Commun. 1994, 10, 1255-1256. (g) Chen, B. C.; Quinlan, S. L.; Stark, D. R.; Reid, J. G.; Audia, W. H.; George, J. G.; Eisenreich, E.; Brundidge, S. P. H.; Racha, S.; Spector, R. H. Tetrahedron Lett. 1995, 36, 7957-7960. (h) Xi, Z.; Agback, P.; Plavec, J.; Sandström, A.; Chattopadhyaya, J. Tetrahedron 1992, 48, 359-370. (i) Chambert, S.; Gautier,-Luneau, I.; Fontecave, M.; Decout, J. L.; J. Org. Chem. 2000, 65, 249-253.

2. Fox, J. J.; Condington, J. F.; Yung, N. C.; Kaplan, L.; Lampen, J. O. J. Am. Chem. Soc. 1958, 80, 5155-5160.

3. Tolstikov, G. A.; Mustafin, A. G.; Gataullin, R. R.; Spirikhin, L. V.; Sultanova, V. S.; Abdrakhmanov, I. B. Russ. Chem. Bull. 1993, 1137-1141.

4. Mustafin, A. G.; Suyundukova, M. V.; Gataullin, R. R.; Spirikhin, L. V.; Abdrakhmanov, I. B.; Tolstikov, G. A. Izv. Akad. Nauk. Ser. Khim 1997, 1420-1421.

5. Hrebabecky, H.; Holy, A. Carbohydr. Res. 1991, 216, 179-186.

6. Robles, R.; Rodrigues, C.; Izquierdo, I; Plaza, M. T.; Mota, A.; de Cienfuegos, L. A. Tetrahedron: Asymmetry 2000, 11, 3069-3077.

7. (a) Shaw, G.; Warrener, R. N. J. Chem. Soc. 1959, 50-55. (b) Ueda, T.; Shibuya, S. Chem. Pharm. Bull. 1970, 18, 1076-1078.

8. Fu, Y. L.; Parthasarathy, R.; Bobek, M. J. Carbohydr. Nucleosides Nucleotides 1978, 5, 7987.

9. (a) Ibrahim, Y. A. Carbohydrate Lett. 1996, 1, 425-432. (b) Ibrahim, Y. A. Carbohydrate Lett. 1996, 2, 189-195. (c) Mansour, A. K.; Ibrahim, Y. A.; Khalil, N. A. S. M. Nucleosides Nucleotides 1999, 18, 2265-2283.

10. Roy Durman, P. Analogues of Nucleic Acid Compounds, Springer Verlag: BerlinHeidelberg-New York, 1970, pp 42-45. 
11. Robins, R. K. Chem. Eng. News 1986, 64, 28.

12. Ibrahim, Y. A.; Abbas, A. A.; Elwahy, A. H. M. Carbohydrate Lett. 1999, 3, 331-338 and references cited therein.

13. (a) Al-Awadi, N. A.; Ibrahim, Y. A.; Kaul, K.; Dib, H. J. Phys. Org. Chem. 2002, 15, 324329. (b) Al-Etaibi, A.; Abdallah, M.; Al-Awadi, N.; Ibrahim, Y.; Hasan, M. J. Phys. Org. Chem. 2004, 17, 49-55.

14. El-Etaibi, A.; Makhseed, S.; Al-Awadi, N. A.; Ibrahim, Y. A. Tetrahedron Lett. 2005, 46, 31-35.

15. (a) Dib, H. H.; Al-Awadi, N. A.; Ibrahim, Y. A.; El-Dusouqui, O. M. E. Tetrahedron 2003, 59, 9455 and references cited therein. (b) Al-Awadi, H.; Ibrahim, M. R.; Dib, H. H.; AlAwadi, N. A.; Ibrahim, Y. A. Tetrahedron 2005, 61, 10507 and references cited therein. (c) Ibrahim, Y. A.; Al-Awadi, N. A.; Kaul, K. Tetrahedron 2001, 57, 7377. 